\title{
Resposta à carta ao editor referente ao artigo "Paralisia obstétrica: De quem é a culpa? Uma revisão sistemática da literatura"
}

\section{Answer to the Letter to the Editor Regarding the Article "Obstetric Paralysis: Who is to Blame? A Systematic Literature Review"}

\author{
José Antonio Galbiatti ${ }^{10}$ Fabrício Luz Cardoso ${ }^{20}$ \\ ${ }^{1}$ Serviço de Ortopedia e Traumatologia, Santa Casa de Misericórdia de \\ Marília, Marília, SP, Brasil \\ 2 Disciplina de Anatomia Humana, Faculdade de Medicina de Marília \\ (FAMEMA), Marília, SP, Brasil \\ 3 Faculdade de Medicina da Universidade de Marília (UNIMAR), Marília, \\ SP, Brasil
}

Rev Bras Ortop 2021;56(3):405-406.

Agradecemos as considerações feita ao artigo. Nosso intuito foi tratar das mudanças que a evolução nos conhecimento médicos e agora apoiados pela revolução digital tem ocasionado em algumas definições clássicas, que se arrastaram por muitos anos. Em nossa discussão, há informações da antiga Medical Insurance Association of America (Associação de Planos de Saúde da América), de janeiro de 1985 a dezembro de 2001, que apresentam dados em que muitas vezes é imputada culpa por imperícia ao médico assistente do parto em casos de paralisia obstétrica. ${ }^{1}$ Desde a publicação do estudo de Jennett et al., ${ }^{2}$ em 1992, acreditamos que, nas publicações dos últimos 20 anos, a forma de encarar a lesão do plexo braquial ao nascimento vem mudando, e, no momento atual, em nossa opinião, essa é uma denominação melhor do que paralisia obstétrica.

Em relação ao título, revisão sistemática de literatura é definida como um estudo secundário, com objetivo de agrupar estudos semelhantes, publicados ou não. Nela avalia-se criticamente a metodologia destes estudos e, quando possível, realizase análise estatística, a metanálise. Por sintetizar dados de estudos primários semelhantes de qualidade científica relevante, é considerada o melhor nível de evidência quando o intuito é a tomada de decisões terapêuticas e condutas em saúde. ${ }^{3,4}$

recebido

15 de Julho de 2020

aceito

16 de Setembro de 2020

Publicado on-line

Março 22, 2021

\author{
Marília Gabriela Palacio Galbiatti ${ }^{3}$
}

Endereço para correspondência Fabrício Luz Cardoso, MD, Av. Monte Carmel, 800, Fragata, Marília, SP, 17519-030, Brasil

(e-mail: fabricioramalhense@gmail.com).

A fim de evitar viés de análise na revisão sistemática, define-se, antes mesmo de a revisão ser iniciada, os métodos de seleção e análise dos dados, num processo rigoroso bem definido. Inicialmente, elabora-se uma hipótese clínica que será o foco do estudo. Em seguida, realiza-se uma ampla busca da literatura com o objetivo de se identificar o maior número possível de estudos relacionados à questão. Após a seleção, aplicam-se critérios para avaliar a qualidade metodológica conforme o delineamento do estudo original. ${ }^{5}$

Dessa forma, concordamos em parte com a crítica apresentada em relação ao título e classificação do estudo. 0 estudo foi denominado "revisão sistemática de literatura", e não apenas "revisão sistemática", por utilizar todos os elementos preconizados na elaboração de uma revisão sistemática clássica, a qual avalia estudos primários: ensaios clínicos randomizados, para a elaboração de uma revisão que reúne exclusivamente artigos de revisão sistemática. Desta forma, utilizaram-se apenas os resultados dessas revisões sistemáticas que são importantes para a medicina baseada em evidências, obtidos dos estudos primários avaliados anteriormente por essas revisões. Tal organização (sistemática) garante a mesma qualidade técnico-científica

(C) 2021. Sociedade Brasileira de Ortopedia e Traumatologia. All rights reserved.

This is an open access article published by Thieme under the terms of the Creative Commons Attribution-NonDerivative-NonCommercial-License, permitting copying and reproduction so long as the original work is given appropriate credit. Contents may not be used for commercial purposes, or adapted, remixed, transformed or built upon. (https://creativecommons.org/ licenses/by-nc-nd/4.0/)

Thieme Revinter Publicações Ltda., Rua do Matoso 170, Rio de Janeiro, RJ, CEP 20270-135, Brazil 
para o nosso estudo, uma vez que avaliaram-se indiretamente vários estudos primários.

Embora sejam mais frequentes as revisões sistemáticas de ensaios clínicos randomizados, na atualidade, há número crescente de revisões desenvolvidas com base em investigações observacionais, como as de caso-controle, transversais, de coorte, os relatos, e as série de casos. Outros delineamentos utilizados são os estudos qualitativos e de avaliação econômica. ${ }^{6}$ Portanto, acreditamos na validade de nosso estudo, cuja metodologia continha a explicação detalhada de como o estudo foi produzido, seguindo rigorosamente os passos de uma boa revisão sistemática: 1) elaboração da hipótese de pesquisa; 2) busca ativa na literatura; 3 ) seleção dos artigos de interesse; 4) extração de dados; 5) avaliação da qualidade metodológica; 6) síntese dos dados/metanálise (única etapa não realizada em nosso artigo); 7) avaliação da qualidade das evidências; e 8) redação e publicação dos resultados. ${ }^{7}$

Nossa revisão demonstra a modificação do direcionamento da principal etiologia da paralisia obstétrica, tirando do médico assistente e de sua equipe o peso preponderante da culpa por imperícia. ${ }^{8,9}$ Atrelado a isso também discutimos que a distocia do ombro não é a principal causa, como descrito anteriormente em alguns estudos. ${ }^{10-16}$

No tocante à quebra de paradigma, é a literatura que tem apontado esse caminho, pois desde Duchenne (1872) e Erb (1874), isto é, por mais de 100 anos, estudos sobre o tema descreviam como princioal culpado o responsável pelo parto (paralisia obstétrica), e nossa intenção é rever de quem é a culpa, pois ela seguramente não é só do médico ou do profissional que fez o parto.

Conflitos de Interesses

Os autores declaram não haver conflito de interesses.

\section{Referências}

1 McAbee GN, Ciervo C. Medical and legal issues related to brachial plexus injuries in neonates. J Am Osteopath Assoc 2006;106(04): 209-212
2 Jennett RJ, Tarby TJ, Kreinick CJ. Brachial plexus palsy: an old problem revisited. Am J Obstet Gynecol 1992;166(6 Pt 1):1673-1676, discussion 1676-1677

3 Atallah AN, Castro AA. Revisão Sistemática e Metanálises, em: Evidências para melhores decisões clínicas. São Paulo: Lemos Editorial; 1998

4 Clarke M, Horton R. Bringing it all together: Lancet-Cochrane collaborate on systematic reviews. Lancet 2001;357(9270): 1728

5 Mulrow CD. Rationale for systematic reviews. BMJ 1994;309 (6954):597-599

6 Centre for Reviews and Dissemination. Systematic reviews: CRD's guidance for undertaking reviews in health care [Internet] YorkCRD, University of York2009 [cited 2020 July 05]. 281 p. Available from: http://www.york.ac.uk/inst/crd/pdf/Systematic Reviews.pdf

7 Galvão T, Pereira M. Revisões sistemáticas da literatura: passos para sua elaboração. Epidemiol Serv Saude 2014;23(01):183-184 Disponível em http://scielo.iec.gov.br/scielo.php?script=sci_arttext\&pid=S1679-49742014000100018

8 Zaki MS, el Sabbagh MH, Aglan MS. Familial congenital brachial palsy: a report of two affected Egyptian families. Genet Couns 2004;15(01):27-36

9 Noble A. Brachial plexus injuries and shoulder dystocia: medicolegal commentary and implications. J Obstet Gynaecol 2005;25 (02):105-107

10 Sandmire HF, DeMott RK. Erb's palsy causation: a historical perspective. Birth 2002;29(01):52-54

11 Krause M, Feige A. [Shoulder dystocia from a legal standpoint]. Z Geburtshilfe Neonatol 2005;209(06):201-209

12 Allen RH. On the mechanical aspects of shoulder dystocia and birth injury. Clin Obstet Gynecol 2007;50(03):607-623

13 Doumouchtsis SK, Arulkumaran S. Are all brachial plexus injuries caused by shoulder dystocia? Obstet Gynecol Surv 2009;64(09): 615-623

14 O'Shea TM, Klebanoff MA, Signore C. Delivery after previous cesarean: long-term outcomes in the child. Semin Perinatol 2010;34(04):281-292

15 Doumouchtsis SK, Arulkumaran S. Is it possible to reduce obstetrical brachial plexus palsy by optimal management of shoulder dystocia? Ann N Y Acad Sci 2010;1205:135-143

16 Anderson JE. Complications of labor and delivery: shoulder dystocia. Prim Care 2012;39(01):135-144 